\title{
COMBINED CLASSIFIERS FOR TIME SERIES SHAPELETS
}

\author{
Ivan S. Mitzev, Nickolas H. Younan \\ Mississippi State University, Mississippi State, MS 39762 \\ ism6@msstate.edu, younandece.msstate.edu
}

\begin{abstract}
Time-series classification is widely used approach for classification. Recent development known as time-series shapelets, based on local patterns from the time-series, shows potential as highly predictive and accurate method for data mining. On the other hand, the slow training time remains an acute problem of this method. In recent years there was a significant improvement of training time performance, reducing the training time in several orders of magnitude. This work tries to maintain low training time- in the range from several second to several minutes for datasets from the popular UCR database, achieving accuracies up to 20\% higher than the fastest known up to date method. The goal is achieved by training small 2,3-nodes decision trees and combining their decisions in pattern that uniquely identifies incoming time-series.
\end{abstract}

\section{KEYWORDS}

Data mining, Time-series shapelets, Combining classifiers

\section{INTRODUCTION}

The time-series shapelets classification method was introduced by Ye and Keogh [1] as a new type of data mining method, that uses the local features of time-series instead of their global. That makes it less sensitive to obstructive noise [1]. This method is successfully applied to a variety of application areas benefiting from its short classification time and high accuracy. Despite its advantages it has a significant disadvantage- a very slow training time. Current research mostly focuses on searching shapelets from all possible combination of time-series derived from a dataset $[1,2]$, keeping the training process relatively slow. A variety of proposals have been introduced to reduce the candidate shapelets $[1,2,4,5]$, but training time is still in the range of hours for some datasets. A newly introduced method [7] shrinks significantly the training time, making the training process to last from portion of a second to several seconds for investigated 45 datasets from UCR collection [9]. Although, this is the fastest up to date training method as of our knowledge, it also maintains high accuracies in compare with other state-of-arts methods [7]. In this paper we introduce a new method that reaches higher accuracies compared with the method from [7], keeping the training time in observable limits. We tested with 24 datasets from [9], the ones with number of classes higher than five. It was found that proposed method outperforms in terms of accuracy the method from [7] for most datasets. The achieved training time is kept low, varying from several seconds to several minutes, depending on a dataset. High accuracy and relatively short training time makes proposed method very competitive to present state-of-arts methods, which lack either accuracy or have huge training time.

Jan Zizka et al. (Eds) : CCSIT, SIPP, AISC, CMCA, SEAS, CSITEC, DaKM, PDCTA, NeCoM - 2016

pp. 173-182, 2016. @ CS \& IT-CSCP 2016

DOI : $10.5121 /$ csit.2016.60116 
The rest of this paper is organized as follows. In section 2 related work is presented. Section 3 describes the proposed method and gives technical details of its implementation. Section 4 discusses achieved results. Finally, section 5 summarizes the proposed method and gives ideas for further work.

\section{RELATED WORK}

Shapelet by definition is a sequence of samples that originate from one of the time-series from a dataset and maximally represent certain class. The classical method of shapelets discovery, known as brute force algorithm [1], employs all possible subsequences from all time-series from the train dataset and treat them as candidate shapelets. To test a candidate shapelet how well separates two classes A and B, all distances between the candidate shapelet and time-series from $\mathrm{A}$ and $\mathrm{B}$ are formed. These distances are ordered into a histogram and the histogram is consecutively split into two parts until the best information gain is achieved. The split point is named optimal split distance and distances below it considered to belong to class A, but above it to class B. If any other candidate shapelet achieves higher information gain, it is selected as shapelet. The process continue until all the candidate shapelets are processed. The method requires vast amount of calculation time. First improvements include subsequence distance early abandon of calculated distances and admissive entropy pruning based on predicted information gain [1]. These improvements reduced the total required time for training, but the reduction was not that significant [6]. Another idea based on the infrequent shapelets, prunes the non-frequent candidate shapelets [4]. More improvements [2] suggests using of so called logical shapelets, that reuse the computation and optimize the search space. Recent approach is based on synthesizing shapelets from random sequences, using particle swarm optimization techniques [6]. A new development in the area [7], vastly improves the training time of the shapelets by pruning candidate segments, which shows similarity in Euclidian distance space. This approach [7] is the fastest up to date as of our knowledge and in terms of accuracy is competitive with the current state-of-arts methods. We selected this method as a reference to proposed method, aiming to achieve similar or better accuracies, maintaining relatively low training time.

\section{PROPOSED METHOD}

Our previous research [6] changes the traditional way of producing shapelets by synthesizing a shapelet from randomly generated sequences using particle swarm optimization (PSO), instead of extracting the shapelets from the original time-series. It finds the shapelet for every pair of classes presented in a dataset, then combines them in a decision tree and find the decision tree that achieves highest accuracy. Producing the most accurate decision tree requires all possible combinations of trees to be tested. That is a slow process for more than four classes and adds additional processing time to traditionally slow training time. For this purpose, only datasets with less than five classes were investigated in [6]. Datasets with more than five classes are processed with the method introduced in this paper. The proposed method utilizes groups of small (up to 4 classes) decision trees, instead of building one big decision tree that contains all classes. When a time-series comes for classification every present tree produce a decision. The decision path taken during classification is present as string of characters. The decision paths from all present trees are combined into decision pattern. Every class from the datasets appears to maintain unique decision pattern. The patterns from training datasets are kept and when new time-series from test dataset comes for classification these patterns are compared with the pattern produced 
from incoming time-series. The incoming time-series is associated with the class, to which its decision pattern mostly match.

\subsection{Training}

\subsubsection{Extracting subsets}

The first step of the training process is to extract subsets of classes out of the original dataset, for which the decision trees will be defined. It is best to have uniform distribution of class indexes into subsets, as it allows non dominant class indexes into the final solution. The maximum amount of subsets $L$ is defined as:

$$
L=K ! /(K-n) ! n !
$$

where, $K$ is the number of all classes in a dataset and $n$ is the number of classes in a subset $n=$ $2,3,4$.

In case the number of classes in the original dataset is relatively high (Fig.1, $K=37$ ), and the subsets consists of four classes for example, the final number of possible subsets combinations becomes 66045, according (1). That is vast amount of subsets and training all of them will defeat the purpose of simplifying the calculations process. Instead of taking all possible combinations we can operate with just limited amount of subsets, obeying the rule of uniform distribution of class indexes as shown on Fig.1. Taking limited amount of subsets will not always fully obey the uniform rule. For example, on Fig. 1 most of classes are present 3 times, but class 21, 29 and 30 are present just two times. Practically, it is enough all class indexes to be present into the subsets and the difference between the number of times classes are present to be no more than one.

\begin{tabular}{|c|c|}
\hline & {$[8,27,29,32]$} \\
& {$[8,19,26,35]$} \\
& {$[4,6,35,37]$} \\
& {$[2,7,11,37]$} \\
& {$[14,20,21,24]$} \\
& {$[21,24,29,33]$} \\
& {$[13,22,32,37]$} \\
& {$[1,8,19,28]$} \\
& {$[4,10,21,25]$} \\
& {$[1,24,26,27]$} \\
& {$[28,31,34,35]$} \\
& {$[4,12,30,36]$} \\
& {$[7,9,26,28]$} \\
& {$[1,5,13,29]$} \\
& {$[2,5,10,25]$} \\
& {$[18,19,22,30]$} \\
& {$[6,13,27,34]$} \\
& {$[3,14,25,34]$} \\
{$[3,37,20,33]$} \\
{$[2,17,31,32]$} \\
{$[9,14,20,22]$} \\
{$[9,10,31,33]$} \\
{$[7,11,15,23]$} \\
{$[3,5,12,30]$} \\
{$[11,16,17,18]$} \\
{$[6,16,18,36]$} \\
{$[12.15 .17 .231$} \\
\hline
\end{tabular}

Fig. 1 Extracted subsets of 4-class combinations from a dataset with class indexes [1..37]. 


\subsubsection{Training decision trees}

Next step of the training process is to create decision trees for extracted subsets. Method from [6] is applied for these subsets as they consist of up to four classes. The method in [6] applies Particle Swarm Optimization (PSO) techniques, treating candidate shapelets as a particles, which form a swarm. To find a shapelet between two classes A and B, swarm of $\mathrm{N}-2$ candidate shapelets is formed. The candidate shapelet represent a random sequence of samples and $\mathrm{N}$ is the length of the time-series in a dataset. Every such sequence has different length, varying from 3 (the smallest meaningful shapelet length) up to $\mathrm{N}$. These potential candidates cover the whole range of possible shapelets lengths. These candidate shapelets compete to each other to find the best solution- a sequence that maximally separates two classes A and B. On every step of the process the candidate shapelet changes its values according to the best overall values in the swarm and candidates best values so far. The fitness function applies functionality similar to the criterions of the brute force algorithm. After calculating the distances between the candidate shapelet and time-series from class A and class B, it builds a histogram of distances and calculate the possible highest information gain. If currently calculated information gain is bigger than information gain assigned so far to the candidate, current values becomes particle's best values. If currently calculated information gain is bigger than information gain of the best candidate shapelet, then current candidate shapelet become the best candidate of overall swarm. Iteration stops when pre-defined number of iterations is achieved or when best information gain from iteration to iteration remains the same.

Described particle searching process is relatively fast, but high number of candidate shapelets may slow down the training process in general. For that, two improvements were introduced. First, compression of the training data is introduced, which according to [7] will not harm the training process, but improves the performance. Second, we introduce the idea that not $\mathrm{N}-2$ candidate shapelets are required, but only 10 will be enough to compete and find the best shapelet among them. As candidate shapelets have different lengths it is important to know which of them to remove from the competition. To find the ten most representative shapelets lengths, we extract from the training dataset only a few time-series per class and train with them. In this partial training process we use $\mathrm{N}-2$ shapelets candidates. The partial training is very fast as just few time-series were used, but it shows well which are the most popular shapelets lengths for certain

subset. Based on these results, we select the 10 most popular shapelets lengths and run the process again.

When all pair of classes in a subset have their shapelets discovered, then all possible variations of decision trees for this subsets are checked and the one that produces the highest accuracy is selected. The accuracy of the decision trees during the training process is checked with timeseries from the training dataset.

\subsubsection{Decision patterns}

An important term in proposed method is the decision path. The decision path is the path taken through the decision tree during decision process. When time-series comes for classification, the distance between shapelet and the time-series is calculated. If such distance is higher than the optimal split distance associated with the shapelet, the process takes the right branch of the tree, if not- the process takes the left branch. When right branch is taken, character " $R$ " is added to the decision path, when the left branch is taken, character " $\mathrm{L}$ " is added to the decision path. The path 
length is equal to the tree depth. An example of possible decision paths are shown on Fig.2. To form a decision pattern the decision paths from all decision trees are concatenated as shown on Fig. 3. For example, if the system consists of 6 decision trees and every tree has two nodes similar to that on Fig.2, then one possible variant of decision patterns is $\{R L, R R, R-, L-L L, R L\}$. During the training process, decision pattern for every time-series from the training dataset is collected. These decision patterns are kept and used during the classification process. Keeping the decision patterns into memory requires certain amount of memory to be allocated during the classification process. For example, "Non.FatalECG.1"[9] dataset contains 1800 train time-series and the chosen pattern length is 836 characters. If we consider that a character is encoded with 1 byte then required memory during classification process is in the vicinity of $1.5 M B$. That is feasible amount for the modern computers, but may cause difficulties in small embedded systems. The direction ("R/L/-") of the decision path currently requires 1 bytes ( 8 bits) of encoding, but it could be optimized to two bits to reduce the required amount of memory, especially in the embedded systems.
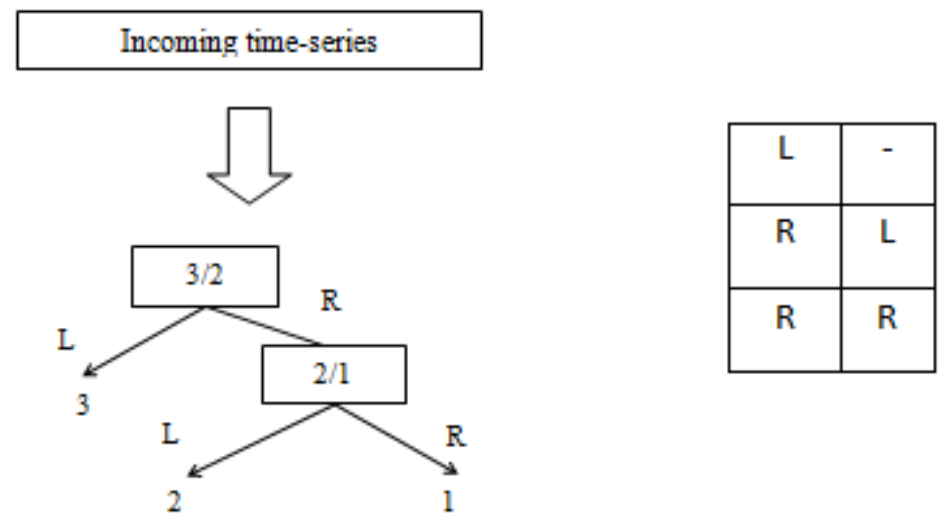

Fig.2 Possible decision paths of the illustrated decision tree obtained after classification of incoming timeseries

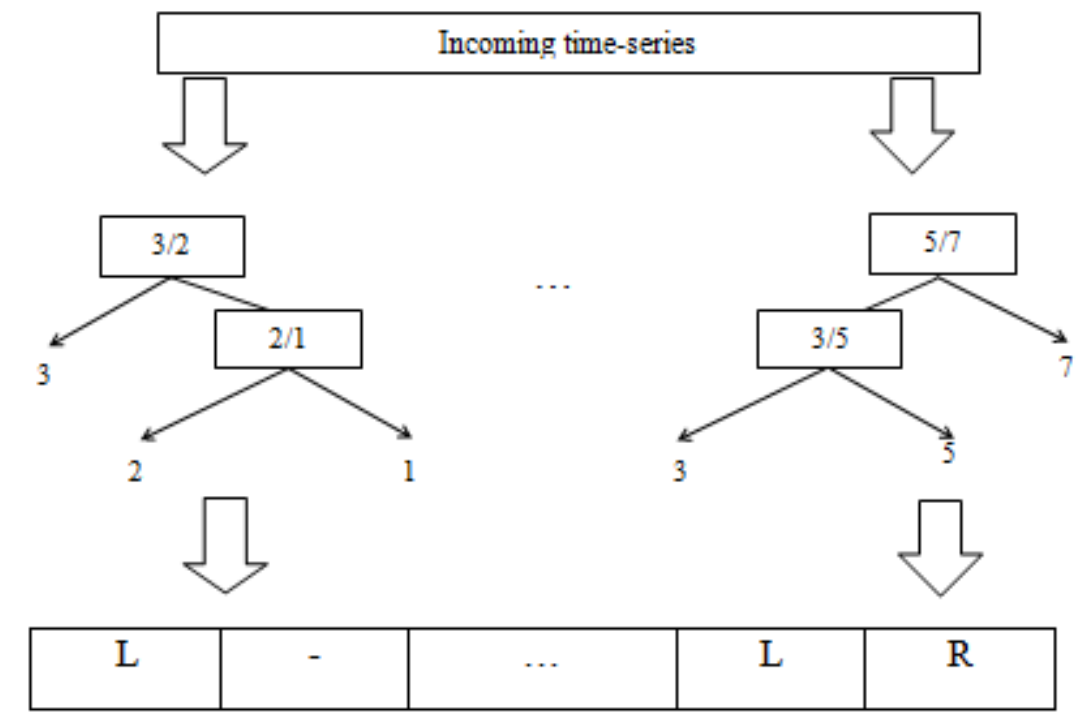

Fig.3 Decision pattern, obtained by combining the decision paths from all subsets' decision trees. 


\subsection{Classification}

When time-series from test dataset comes for classification a decision pattern is created for that time-series. This pattern is compared with the patterns produced during the training process. Comparison process is very simple. The two decision patterns strings are compared character by character in place and the comparison coefficients is equal to the number of characters that coincide by place and value, divided by the length of the decision string as shown on Fig. 4. After all the comparison coefficients are collected we keep only those which are above certain threshold of 0.98. The idea of this classification is that time-series from the same class will produce similar decision patterns, but decision patterns from different classes will differ a lot. The incoming time-series is identified as class to which it has closest decision pattern. In certain cases more than one class index show similar pattern to the investigates time-series. In such cases the classification process assign the incoming time-series to the class, that has majority of decision patterns closest to the incoming time-series decision pattern.

\begin{tabular}{|l|l|l|l|l|l|l|l|l|l|}
\hline R & - & L & L & R & - & L & L & L & R \\
\hline R & L & L & L & L & - & L & R & L & L \\
\hline
\end{tabular}

Fig. 4 Comparison between decision patterns of two time-series. Six out of ten characters coincide by place and value, therefore the comparison coefficients is $6 / 10=0.6$.

\section{EXPERIMENTAL RESULTS}

The project implementation uses C\# and .NET Framework 4.0. Time performance measurements were produced with a System.Diagnostics.StopWatch .NET class. In our experiments we used a PC with the following parameters: CPU: Intel Core i7, 2.4GHz; RAM: 8 GB; 64-bit Windows 7 OS. We selected datasets from the UCR collection [9] with a number of classes higher than five (Table 1) as for datasets with fewer classes applying proposed method is meaningless. Table 1 shows parameters of the used datasets. We used method from [7] as a reference method. It produces the fastest training time as of our knowledge and accuracies that outperform most of state-of-arts method for the moment. We downloaded the Java implementation of the proposed method from [10] and ran it on the same hardware as proposed method. Reference method requires to specify threshold $p$ and aggregation ratio $r$. We kept these value the same as in [7] to maintain the highest accuracy. Table 3 shows the results of both methods in terms of training time and accuracies they produce. In 18 out of 24 cases the proposed method outperforms the reference method in terms of accuracy, where the improvements vary from $2 \%$ up to $23 \%$, where in six of these cases the improvement is above $10 \%$. In the rest, 3 cases differ less than $1.0 \%$ and we consider that both method perform equally in this cases. Only in 3 cases the reference method outperform the proposed method in terms of accuracy, but the difference is less than $2 \%$. Although the reference method shows better training times, the proposed method maintains an observable training time- varying from several seconds up to several minutes $(\sim 15 \mathrm{~min}$. for Non.FatalECG.1) for datasets that have long time-series and higher number of time-series in a train datasets (uWave.X, uWave.Y, uWave.Z).

Proposed method uses the decision patterns from all time-series in the training datasets thus, in some cases the classification process may slow done due to high number of time-series in the train dataset (Non.FatalECG.1, Non.FatalECG.2). In some cases the length of the decision pattern 
could be relatively long (Adiac- 1473, Non.FatalECG.1- 836) which may also influence the classification time. To investigate this issue we have measured the averaged classification time per time-series. Table 2 represents the results. In the most heavy case (Non.FatalECG.1) when the number time-series is 1800 and the decision pattern string length is 836 characters the classification time is above 200 milliseconds. The length of the decision pattern may very as shown on Table 3. For datasets, such as "Beef", which consist of 5 classes, the number of subsets is limited to 10 when constructed of 3 class indexes or to 5 when constructed of 4 class indexes. In this case to achieve better accuracy, combination of all possible trees up to 4 indexes are taken. On the other hand, datasets with more class indexes have more varieties to choose from. In the case of " 50 Word" dataset, which contain 50 class indexes, the total amount for combinations for two-classes decision tree is 1225 . We selected 497 of them based on the principle from 3.1.1 and the total length of the decision pattern become 994 characters. Rising the number of characters in the decision pattern in all investigated cases increased the accuracy in general. Although, it appears that there is certain limit of characters above which the accuracy does not increase and even may decrease as shown on Table 4.

Table 1. Used datasets from UCR database.

\begin{tabular}{|c|c|c|c|}
\hline Dataset & $\begin{array}{c}\text { Number of } \\
\text { Classes }\end{array}$ & $\begin{array}{c}\text { Number of } \\
\text { time-series } \\
\text { in the } \\
\text { train/test } \\
\text { dataset }\end{array}$ & $\begin{array}{c}\text { Time-series } \\
\text { length }\end{array}$ \\
\hline Beef & 5 & $30 / 30$ & 470 \\
\hline Haptics & 5 & $155 / 308$ & 1092 \\
\hline OsuLeaf & 6 & $200 / 242$ & 427 \\
\hline Symbols & 6 & $25 / 995$ & 398 \\
\hline synthetic. & 6 & $300 / 300$ & 60 \\
\hline Fish & 7 & $175 / 175$ & 463 \\
\hline InlineSkate & 7 & $100 / 550$ & 1882 \\
\hline Lighting7 & 7 & $70 / 73$ & 319 \\
\hline MALLAT & 8 & $55 / 2345$ & 1024 \\
\hline uWave.X & 8 & $896 / 3582$ & 315 \\
\hline uWave.Y & 8 & $896 / 3582$ & 315 \\
\hline uWave.Z & 8 & $896 / 3582$ & 315 \\
\hline MedicalImages & 10 & $381 / 760$ & 99 \\
\hline Cricket X & 12 & $390 / 390$ & 300 \\
\hline Cricket Y & 12 & $390 / 390$ & 300 \\
\hline Cricket Z & 12 & $390 / 390$ & 300 \\
\hline FaceAll & 14 & $560 / 1690$ & 131 \\
\hline FacesUCR & 14 & $200 / 2050$ & 131 \\
\hline SwedishLeaf & 15 & $500 / 625$ & 128 \\
\hline WordsS. & 25 & $267 / 638$ & 270 \\
\hline Adiac & 37 & $390 / 391$ & 176 \\
\hline Non.FatalECG.1 & 42 & $1800 / 1965$ & 750 \\
\hline Non.FatalECG. 2 & 42 & $1800 / 1965$ & 750 \\
\hline 50words & 50 & $450 / 455$ & 270 \\
\hline
\end{tabular}


Table 2. Averaged classification times produced by proposed method.

\begin{tabular}{|l|r|l|r|}
\hline \multicolumn{1}{|c|}{ Dataset } & $\begin{array}{c}\text { Classification } \\
\text { Time, [msec] }\end{array}$ & \multicolumn{1}{c|}{ Dataset } & $\begin{array}{c}\text { Classification } \\
\text { Time, [msec] }\end{array}$ \\
\hline Beef & 0.38 & MedicalImages & 4.27 \\
\hline Haptics & 1.05 & Cricket X & 11.48 \\
\hline OsuLeaf & 1.67 & Cricket Y & 9.07 \\
\hline Symbols & 0.72 & Cricket Z & 9.07 \\
\hline synthetic. & 1.81 & FaceAll & 10.06 \\
\hline Fish & 3.97 & FacesUCR & 3.97 \\
\hline InlineSkate & 4.14 & SwedishLeaf & 16.03 \\
\hline Lighting7 & 2.17 & WordsS. & 17.75 \\
\hline MALLAT & 2.11 & Adiac & 64.56 \\
\hline uWave.X & 3.98 & Non.FatalECG.1 & 223.52 \\
\hline uWave.Y & 5.87 & Non.FatalECG.2 & 195.99 \\
\hline uWave.Z & 3.84 & 50words & 66.77 \\
\hline
\end{tabular}

Table 3. Experimental results presenting accuracies and training times of the proposed and reference method.

\begin{tabular}{|l|r|r|r|r|r|c|}
\hline \multirow{2}{*}{ Dataset } & & \multicolumn{3}{c|}{ Proposed method } & \multicolumn{2}{c|}{ Reference method } \\
\cline { 3 - 7 } & $\begin{array}{c}\text { Comp. } \\
\text { Rate }\end{array}$ & $\begin{array}{c}\text { Pattern } \\
\text { Length }\end{array}$ & $\begin{array}{c}\text { Train } \\
\text { Tim } \\
\text { e, } \\
{[\mathbf{s e c}]}\end{array}$ & $\begin{array}{c}\text { Accuracy, } \\
{[\mathbf{\%}]}\end{array}$ & $\begin{array}{c}\text { Train } \\
\text { Tim } \\
\text { e, } \\
{[\mathbf{s e c}]}\end{array}$ & $\begin{array}{c}\text { Accuracy, } \\
{[\%]}\end{array}$ \\
\hline Beef & 0.125 & 70 & 4.15 & $\mathbf{5 2 . 2 1}$ & 0.05 & 48.89 \\
\hline Haptics & 0.500 & 20 & 70.66 & $\mathbf{3 9 . 3 9}$ & 1.69 & 34.56 \\
\hline OsuLeaf & 0.125 & 150 & 55.84 & $\mathbf{7 6 . 9 9}$ & 0.14 & 53.31 \\
\hline Symbols & 0.250 & 150 & 7.87 & $\mathbf{9 4 . 2 0}$ & 0.05 & 82.48 \\
\hline synthetic. & 0.250 & 150 & 125.58 & 98.88 & 0.06 & 98.44 \\
\hline Fish & 0.250 & 287 & 102.51 & $\mathbf{9 0 . 8 5}$ & 0.15 & 75.05 \\
\hline InlineSkate & 0.125 & 245 & 78.57 & 39.57 & 0.56 & 39.88 \\
\hline Lighting7 & 0.500 & 245 & 42.52 & $\mathbf{7 5 . 7 9}$ & 0.39 & 65.30 \\
\hline MALLAT & 0.125 & 280 & 42.87 & $\mathbf{9 2 . 8 5}$ & 0.10 & 90.77 \\
\hline uWave.X & 0.250 & 117 & 559.22 & 75.32 & 4.37 & $\mathbf{7 6 . 4 5}$ \\
\hline uWave.Y & 0.250 & 168 & 594.66 & 65.12 & 3.33 & $\mathbf{6 6 . 7 2}$ \\
\hline uWave.Z & 0.125 & 117 & 508.93 & 66.30 & 1.89 & $\mathbf{6 7 . 4 8}$ \\
\hline Med.Images & 0.500 & 240 & 139.47 & $\mathbf{7 1 . 2 7}$ & 0.58 & 67.68 \\
\hline Cricket X & 0.250 & 471 & 267.66 & $\mathbf{7 7 . 7 8}$ & 0.61 & 68.63 \\
\hline Cricket Y & 0.250 & 408 & 198.58 & $\mathbf{7 9 . 1 4}$ & 0.50 & 64.01 \\
\hline Cricket Z & 0.250 & 414 & 184.38 & $\mathbf{7 5 . 2 9}$ & 0.66 & 68.21 \\
\hline FaceAll & 0.500 & 342 & 167.02 & $\mathbf{7 5 . 4 2}$ & 1.25 & 71.63 \\
\hline FacesUCR & 0.500 & 330 & 36.97 & $\mathbf{9 0 . 5 6}$ & 0.32 & 84.61 \\
\hline SwedishLeaf & 0.500 & 519 & 342.27 & $\mathbf{9 1 . 1 4}$ & 0.34 & 85.60 \\
\hline WordsS. & 0.250 & 600 & 28.48 & $\mathbf{6 5 . 4 6}$ & 0.29 & 60.92 \\
\hline
\end{tabular}




\begin{tabular}{|l|c|c|r|r|r|l|}
\hline Adiac & 0.500 & 1473 & 514.63 & $\mathbf{7 3 . 6 5}$ & 0.27 & 55.67 \\
\hline Non.FatalECG.1 & 0.250 & 836 & 878.18 & $\mathbf{8 5 . 0 1}$ & 6.90 & 80.93 \\
\hline Non.FatalECG.2 & 0.125 & 836 & 349.32 & $\mathbf{8 9 . 1 9}$ & 4.67 & 86.34 \\
\hline 50words & 0.250 & 994 & 58.15 & 68.79 & 0.35 & 68.06 \\
\hline
\end{tabular}

Table 4. Accuracy dependency from decision pattern length for "Lighting7"( 7 classes) dataset.

\begin{tabular}{|c|r|c|}
\hline $\begin{array}{c}\text { Decision Pattern Length (number } \\
\text { of trees x number of classes in } \\
\text { tree ) }\end{array}$ & Training Time, [sec] & Accuracy, [\%] \\
\hline $21 \times 2$ & 4.09 & 61.64 \\
\hline $35 \times 3$ & 16.28 & 71.23 \\
\hline $35 \times 4$ & 30.90 & 71.68 \\
\hline $35 \times 4+35 \times 3$ & 42.52 & 75.79 \\
\hline $35 \times 4+35 \times 3+21 \times 2$ & 44.29 & 73.97 \\
\hline
\end{tabular}

\section{CONCLUSION AND FUTURE WORK}

This paper proposes a new method for time-series shapelets classification, which demonstrate higher accuracies than produced by fastest known state-of-arts method for most of the investigated cases. As well it keeps an observable time for training, varying from several seconds to several minutes.

As future work we will focus on improving the training time even further, applying parallel processing based calculations (utilizing .NET Parallel.ForEach) that employ all possible processor's cores on certain machine. This technology could be successfully applied on variety of places in proposed method where the calculations from current stage are independent from each other, namely: iteration steps of the PSO algorithm for shapelets discovery; the comparison between incoming time-series pattern and available decision patterns.

\section{REFERENCES}

[1] L. Ye and E. Keogh, "Time series shapelets: a new primitive for data mining," in Proceedings of the 15th ACM SIGKDD International Conference on Knowledge Discovery and Data Mining, 2009.

[2] A. Mueen, E. Keogh, and N. Young, "Logical-shapelets: an expressive primitive for time series classification," in Proceedings of the 17th ACM SIGKDD International Conference on Knowledge Discovery and Data Mining, 2011.

[3] T. Rakthanmanon and E. Keogh, "Fast shapelets: A scalable algorithm for discovering time series shapelets," Proceedings of the 13th SIAM International Conference on Data Mining, 2013.

[4] He1 Q., Dong Z., Zhuang F., Shang T., Shi Z., "Fast Time Series Classification Based on Infrequent Shapelets", 2012 11th International Conference on Machine Learning and Applications, 2012

[5] J. Yuan, Z. Wang, H. Meng, „A discriminative Shapelets Transformation for Time Series Classification“, International Journal for Pattern Recognition and Artificial Intelligence, Vol. 28, No. 6, 2014. 
[6] I. Mitzev, N. Younan, (2015), "Time Series Shapelets: Training Time Improvement Based on Particle Swarm Optimization", 7th International Conference on Machine Learning and Computing, March 2015

[7] J. Grabocka, M. Wistuba, L. Schmidt-Thieme, "Scalable Discovery of Time-Series Shapelets", arXiv:1503.03238 [cs.LG], March 2015

[8] J. Lines, L. Davis, J. Hills, A. Bagnall, "A Shapelet Transform for Time Series Classification", Proceedings of the 18th ACM SIGKDD International Conference on Knowledge Discovery and Data Mining, 2012

[9] E. Keogh, Q. Zhu, B. Hu, H. Y., X. Xi, L. Wei, and C. A. Ratanamahatana, "The UCR Time Series Classification/Clustering Homepage," www.cs.ucr.edu/ eamonn/time_series_data

[10] J. Grabocka, M. Wistuba, L. Schmidt-Thieme, Source Code and Executables for Scalable Discovery of Time-Series Shapelets algorithm, https://www.dropbox.com/sh/btiee2pyn6a989q/AACDfzkkpdYPmgw7pgTgUoeYa

[11] P.Senin, S.Malinchik, "SAX-VSM: Interpretable Time Series Classification Using SAX and Vector Space model”, Data Mining (ICDM), 2013 IEEE 13th International Conference, 2013

[12] D. Gordon, D. Hendler, L. Rokach, "Fast Randomized Model Generation for Shapelet-Based Time Series Classification", arXiv:1209.5038 [cs.LG], 2012

[13] J. Grabocka, N. Schilling, M.Wistuba, L.Schmidt-Thieme, "Learning Time-Series Shapelets", KDD’14, August 24-27, 2014, NY, USA, 2014

\section{AUTHORS}

Ivan S. Mitzev is currently $\mathrm{PhD}$ candidate of Electrical and Computer Engineering at Mississippi State University. He received his M.S. degree of Electrical Engineering from Mississippi State University in 2010. His research interests include software development, pattern recognition and bio-medical signal processing.

Nicolas H. Younan is currently the Department Head and James Worth Bagley Chair of Electrical and Computer Engineering at Mississippi State University. He received the B.S. and M.S. degrees from Mississippi State University, in 1982 and 1984, respectively, and the Ph.D. degree from Ohio University in 1988. Dr. Younan's research interests include signal processing and pattern recognition. He has been involved in the development of advanced signal processing and pattern recognition algorithms for data mining, data fusion, feature extraction and classification, and automatic target

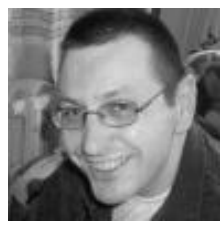
recognition/identification.

Dr. Younan has published over 250 papers in refereed journals and conference proceedings, and book chapters. He has served as the General Chair and Editor for the 4th IASTED International Conference on Signal and Image Processing, Co-Editor for the 3rd International Workshop on the Analysis of MultiTemporal Remote Sensing Images, Guest Editor, Pattern Recognition Letters, and JSTARS, and Co-Chair, Workshop on Pattern Recognition for Remote sensing (2008-2010). He is a senior member of IEEE and a member of the IEEE Geoscience and Remote Sensing society, serving on two technical committees: Image Analysis and Data Fusion, and Earth Science Informatics (previously Data Archive and Distribution). He also served as the Vice Chair of the International Association on Pattern Recognition (IAPR) Technical Committee 7 on Remote Sensing (2008-2010), and Executive Committee Member of the International Conference on High Voltage Engineering and Applications(2010-2014). 